\title{
Avaliação do Dimensionamento de Símbolos Pictóricos em Mapas Indoor com Perspectiva Egocêntrica para Aplicação em Sistemas de Realidade Aumentada
}

\author{
Evaluation of the Dimensioning of Pictorial Symbols in Internal Maps with an Egocentric Perspective \\ for Application in Augmented Reality Systems
}

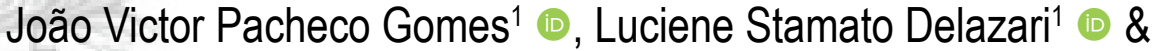 Marcio Augusto Reolon Schmidt ${ }^{1,2}$

\author{
${ }^{1}$ Universidade Federal do Paraná, Programa de Pós-Graduação em Ciências Geodésicas, Centro Politécnico, Curitiba, PR, Brasil \\ Universidade Federal de Uberlândia, Programa de Pós Graduação em Engenharia Civil, Faculdade de Engenharia Civil, \\ Uberlândia, MG, Brasil \\ E-mails: joaovictorpac@gmail.com; luciene@ufpr.br; marcio.schmidt@ufu.br
}

\section{Resumo}

A aplicação das leis da Gestalt, como, por exemplo, a separação figura-fundo e o tamanho (dimensões) de representações gráficas podem melhorar a percepção dos símbolos pictóricos. Deste modo, objetivo do artigo é verificar se a variação em tamanho possibilita ao usuário perceber a profundidade e distância dos símbolos para aplicação em Realidade Aumentada (RA) de ambientes internos (indoor), com base na vista em perspectiva egocêntrica. A pesquisa aplicada utiliza um conjunto de símbolos pictóricos alterados para a representação em RA simulada, considerando as leis da Gestalt. Posteriormente, realizaram-se testes empíricos no contexto do ambiente indoor em que símbolos com variações de tamanho foram dispostos em três cenários com diferenças na iluminação do ambiente e contraste figura-fundo, avaliando a percepção do entrevistado em relação as variações de distância. Os resultados obtidos no experimento indicaram que a variação no tamanho dos símbolos permite obter a noção de proximidade e distanciamento, mas pode sofrer influência do ambiente devido ao grau de contraste (claro-escuro). Demonstrou-se ainda, que para sistemas de RA indoor, a avaliação do tamanho dos símbolos permite compreender a relação de proximidade ou distanciamento entre o usuário e os pontos de referência.

Palavras-chave: Símbolos pictóricos; Ambiente indoor; Realidade aumentada

\begin{abstract}
The application of Gestalt laws, such as, for example, the separation of background figure and the size (dimensions) of graphic representations, can improve the perception of pictorial symbols. In this way, the objective of the article is to verify if the variation in size allows the user to perceive the depth and distance of the symbols for application in Augmented Reality (AR) of indoor environments (indoor), based on the egocentric perspective view. Applied research uses a set of altered pictorial symbols for representation in simulated AR, considering the laws of Gestalt. Subsequently, empirical tests were carried out in the context of the indoor environment in which symbols with variations in size were arranged in three scenarios with differences in ambient lighting and figure-to-background contrast, assessing the interviewee's perception of distance variations. The results obtained in the experiment indicated that the variation in the size of the symbols allows one to obtain the notion of proximity and distance, but may be influenced by the environment due to the degree of contrast (light-dark). It was also demonstrated that for indoor AR systems, the evaluation of the size of the symbols allows to understand the relationship of proximity or distance between the user and the reference points.
\end{abstract}

Keywords: Pictorial symbols; Indoor environment; Augmented reality 


\section{Introdução}

Em geral, o crescimento populacional no espaço urbano acelera o processo de verticalização e o aumento na complexidade das edificações nas grandes cidades (Vanclooster, Van De Weghe \& De Maeyer 2016; Wang $\&$ Niu 2018). A construção de edifícios com escritórios voltados à atividade profissional diversa ou, até mesmo, centros comerciais de grandes dimensões, denotam a complexidade inerente a estes espaços que tendem a comportar um alto fluxo de pessoas. O termo indoor denota o interior dessas edificações que se caracterizam por ambientes eminentemente fechados, ou seja, edificações que contém pouco ou nenhum contato com o ambiente externo e nos quais o ser humano dispensa a maior parte do seu tempo. A representação das informações locacionais adquire tanta importância nos espaços indoor quanto nos espaços externos, com base na necessidade de possibilitar às pessoas uma orientação espacial segura e eficaz (Ilkovičová, Erdélyi \& Kopáćik 2014; Liu et al. 2016).

A representação do espaço indoor deve considerar a existência de características estruturais que o distinguem do espaço externo. Como exemplo, cita-se a existência de paredes que o delimitam e sugerem sua separação em relação ao espaço externo (Biehler \& Simon 2011) e a existência, em muitos casos, de mais de um pavimento. A Cartografia estuda questões acerca da conectividade de subespaços, horizontalidade de corredores, e os agentes conectivos de transição vertical para fins de representação. Diferentes soluções têm sido objetos de estudo, como os modelos de representações bidimensionais (2D), tridimensionais (3D) e em Realidade Aumentada (Liu et al. 2016; Nossum 2013), a Figura 1 apresenta respectivamente esses modelos de representação.

A realidade aumentada (RA) é uma forma de representação que permite trabalhar em conjunto elementos virtuais e a representação do espaço concreto-real, exigindo baixo nível de abstração da realidade (Al Delail et al. 2013). A possibilidade de aplicação dos dispositivos móveis (os smartphones) como interface para visualização da RA, pode facilitar a orientação espacial pessoal a partir de um dispositivo de uso pessoal e diário (Dick et al. 2016). As pesquisas que buscam implementar a RA como tecnologia de representação de informações cartográficas indoor, têm destacado a importância de estudos relativos ao uso da linguagem cartográfica (Halik \& Medyńska-Gulij 2016; Li et al. 2019).

Em decorrência dos poucos estudos desenvolvidos no contexto da linguagem cartográfica para RA, as pesquisas desenvolvidas por Halik (2012); Halik \& Medyńska-Gulij (2016); e Stanek \& Friedmannova (2010) se destacam

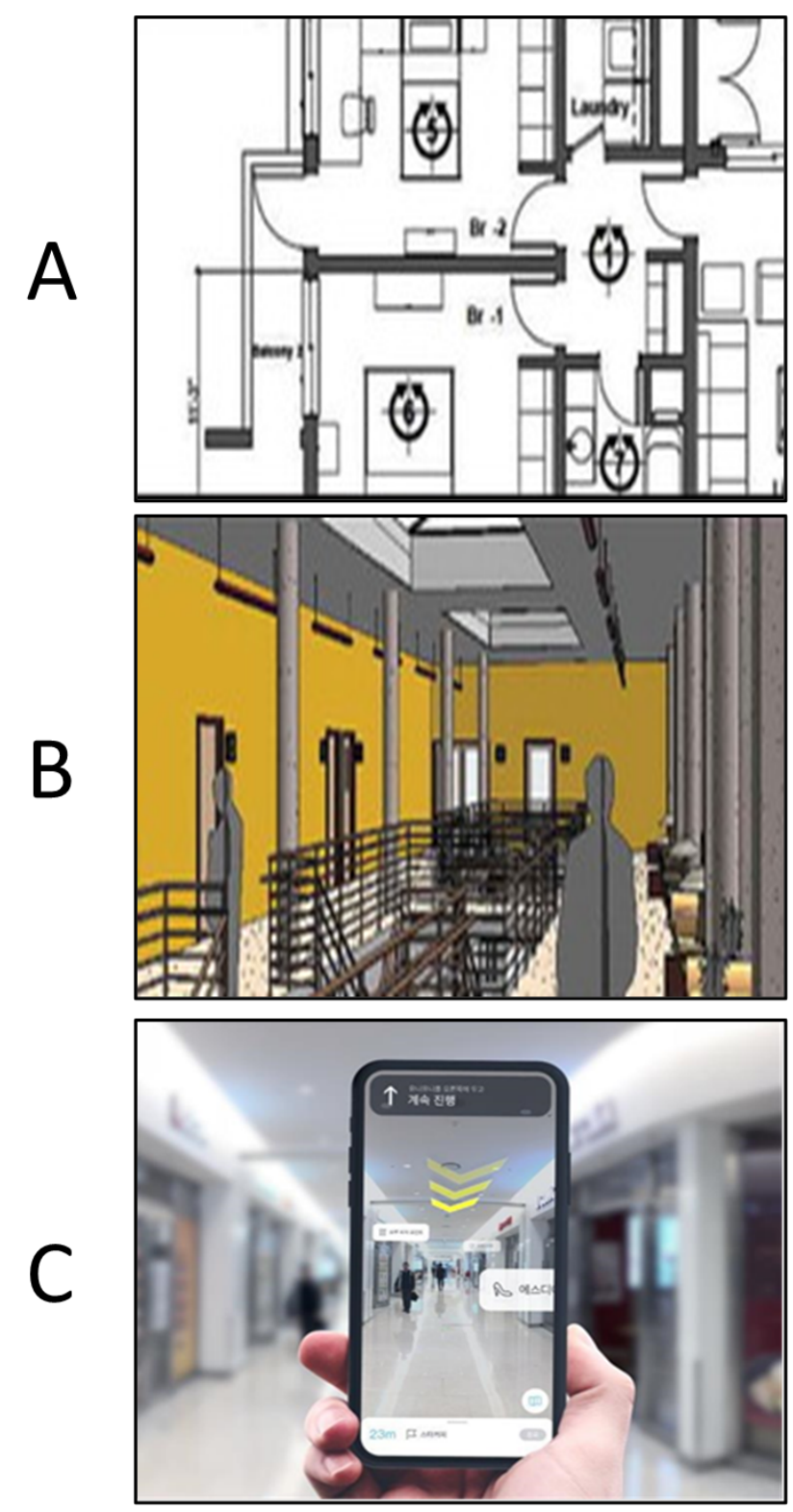

Figura 1 A. Representação 2D; B. Representação 3D; C. Representação em RA (Adaptado de Chen \& Clarke 2020; Naver 2020).

por apresentar diretrizes para a construção e aplicação de uma simbologia. Segundo os autores, em sistemas de RA as informações qualitativas devem ser apresentadas, preferencialmente, através de símbolos pontuais dispostos na cena (Halik 2012, Stanek \& Friedmannova 2010). Por esse motivo, considerando a qualidade de interpretação da informação por parte do usuário, os autores indicam a aplicação da simbologia pictórica em sistemas de RA para representações cartográficas (Halik \& Medyńska-gulij 2016; Stanek \& Friedmannova 2010). 
Os símbolos pictóricos tendem a assemelhar-se do elemento representativo do mundo real (MacEachren 1995; Massironi 2015; Wong 2001), a partir de semelhanças físicas/miméticas que diminuem o nível de abstração necessário para sua interpretação (Fiori \& Lucena 2020). A eficiência do símbolo pictórico pode ser avaliada a partir da rapidez no processo de percepção e compreensão da informação pelo leitor. A Figura 2 apresenta um exemplo de aplicação do símbolo pictórico, geralmente utilizado para indicar a posição de sanitários em prédios públicos, observa-se que a semelhança com a forma masculina e feminina é obtida sem apresentar riqueza de detalhes.

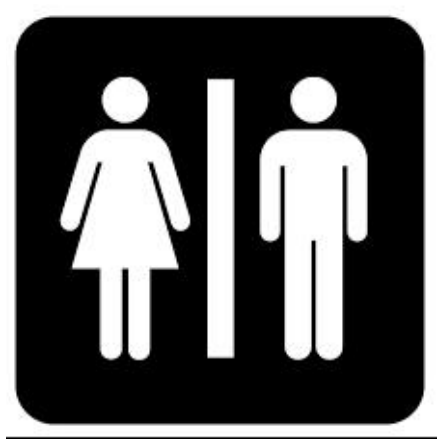

Figura 2 Exemplo de símbolo pictórico

Fonte: Pixabay 2019.

A decodificação da mensagem transmitida pelo símbolo pictórico ocorre com a identificação de traços socioculturais presentes na sua composição e que permitem ao leitor realizar associação no processo interpretativo e compreender o seu significado dentro do contexto no qual está inserido (Korpi \& Ahonen-Rainio 2010, 2015; Wong 2001). Portanto, a representação pictórica depende do conhecimento cultural por parte do receptor. O processo de decodificação da informação a partir do conhecimento cultural, é parte de uma teoria mais abrangente conhecida como teoria da Gestalt. A Gestalt se refere à compreensão do objeto por diferentes atributos, sobretudo a forma, fazendo com que esta teoria também seja conhecida como teoria perceptiva da forma (Andrade 2014; Hogg 2012; Santil 2008).

Para a simbologia pictórica, a teoria da Gestalt proporciona um embasamento para a construção de símbolos com maior capacidade de identificação e reconhecimento. Os símbolos pictóricos devem ser reconhecidos em sua totalidade e não a partir da identificação das pequenas partes que o compõe (Andrade 2014). No entanto é possível destaca-los do todo (Andrade 2014, Santil 2008). O autor se preocupa em representar o espaço concreto-real na tela do dispositivo móvel. Aliás, o espaço concreto-real serve como suporte ao processo perceptivo do usuário em relação às informações cartográficas dispostas na tela (Andrade 2014). Ressaltar o símbolo do espaço concreto-real é o processo de segregação figura-fundo da Gestalt em que, com base em princípios de heterogeneidade, contorno, orientação e tamanho relativo é possível separar objetos (símbolos) de não objetos (fundo), conforme aponta MacEachren (1995).

Estudos que abordam a aplicação de símbolos pictóricos, construídos com base nas leis da Gestalt para representação cartográfica em sistemas de RA ainda são incipientes, sobretudo no contexto dos dispositivos móveis com tela reduzida, visão egocêntrica e vista perspectiva. Nesse aspecto, os estudos acerca da representação 2D e 3D têm embasado as representações em RA. Haeberling (2002) apresenta aspectos de uma representação cartográfica em perspectiva, que podem ser aplicadas nesse contexto, no que se refere especificamente ao 2,5D (duas dimensões e meio ou pseudo tridimensão).

Para Kubíček et al. (2017) os dispositivos móveis que permitem a integração do usuário com o ambiente virtual, aplicam um efeito visual de profundidade permitindo que o usuário tenha uma sensação de perspectiva ao visualizar a representação (Figura 3). As dicas de profundidade (Kubíček et al. 2017; Liu et al. 2016) foram apresentadas por Kraak (1988) como taxonomia de pistas (depth cues), como, por exemplo, o gradiente de textura que representa linhas mais grossas para elementos no primeiro plano e linhas mais finas para objetos mais ao fundo, como pode ser observado na Figura 3. As taxonomias de pistas são fornecidas através dos símbolos empregados na representação do ambiente, e que podem estar na forma geral ou em detalhe dependendo do distanciamento ou proximidade da informação retratada.

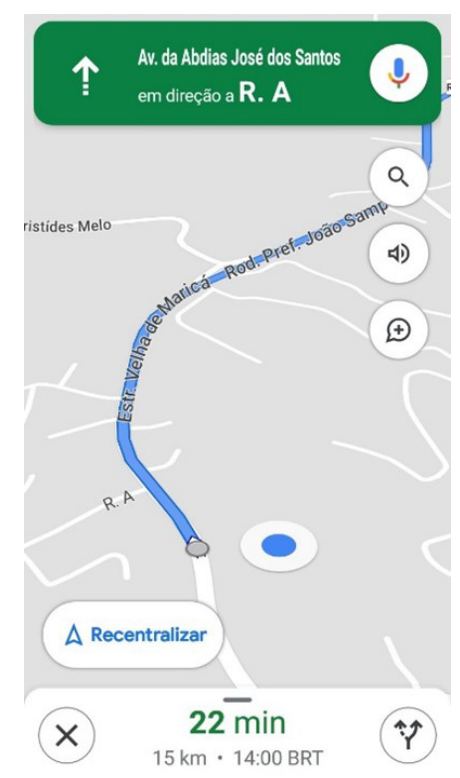

Figura 3 Exemplo de representação com efeito de profundidade. 
Um aspecto importante que Kraak (1988) ressalta em relação a simbologia, é que a representação visual de cada símbolo não possui um tamanho fixo, de modo que a aparência dos símbolos pode variar a partir da escala, adquirindo maior ou menor tamanho para representar sua configuração espacial de proximidade e/ou distanciamento. Segundo Halik (2012), em um sistema de RA, a noção de profundidade de campo do usuário está diretamente relacionada a variação do tamanho dos símbolos, que são percebidos a partir da dinâmica do distanciamento. Desta forma, alguns aspectos gráficos dos símbolos podem variar, como a tonalidade, forma, tamanho, largura das linhas, entre outros.

Os estudos apresentados, até o momento referemse a experimentos de representação em RA para espaços externos. No entanto, o recurso tem sido pouco explorado em espaços internos (Nossum 2013; Vanclooster, Van De Weghe \& De Maeyer 2016; Rantakari et al. 2017). Com aumento da complexidade das edificações o mapeamento indoor não é mais uma tendência (Biehler \& Simon 2011; Schabus, Scholz \& Lampoltshammer 2015), mas sim uma necessidade, com base na importância crescente de possibilitar às pessoas uma navegação segura nesses ambientes (Ilkovičová, Erdélyi \& Kopáčik 2014). Assim, são necessários experimentos que tenham como objetivo verificar se os símbolos pictóricos, aplicados com base nas leis da Gestalt, podem proporcionar ao usuário a noção de distanciamento ou proximidade em sistemas de RA indoor.

Deste modo, parte-se do princípio que para as representações tridimensionais, a noção de distanciamento ou proximidade podem ser obtidas através dos símbolos pictóricos com base nas leis da Gestalt. Então, ao aplicar as mesmas leis na representação de RA em um ambiente indoor, o efeito de profundidade e distanciamento será percebido da mesma forma. A validação da hipótese é realizada por meio de um teste com usuários em que duplas de símbolos pictóricos - com tamanhos relativos diferentes entre si - são apresentados em uma representação de RA simulada em espaço indoor. A pesquisa avalia a capacidade perceptiva do usuário para estimar a proximidade em relação aos pontos de referência representados através dos símbolos.

\section{Materiais e Métodos}

Este estudo se baseia nas pesquisas de Haeberling (2002); Halik (2012); Halik \& Medyńska-Gulij (2016); e Stanek \& Friedmannova (2010) e visa identificar a influência da dimensionalidade dos símbolos pictóricos na estimativa das distâncias do espaço real-concreto a partir de representação gráfica em RA. Contudo, os autores ainda não possuem uma pesquisa mais aprofundada, efetiva, em espaços indoor.
A pesquisa considera que a relação de tamanho entre os símbolos, somada a posição do ângulo de visada no contexto de uso permitem que o usuário estime a distância relativa no ambiente indoor. $\mathrm{O}$ estudo desenvolve um experimento em espaços internos de edifícios públicos, nos quais os participantes foram expostos a um conjunto de símbolos pictóricos projetados em áreas de circulação comum, tendo que indicar que símbolo estava mais próximo a ele (o pesquisado).

A realização do teste procurou avaliar a percepção dos símbolos pictóricos a partir do uso de imagens estáticas com o ponto de vista em perspectiva egocêntrica do espaço. Os símbolos deveriam estar sempre na mesma posição e próximos ao ângulo de visada do observador. Tal abordagem é necessária nesse estágio da pesquisa, pois o movimento de um dispositivo de teste (no caso, levantar ou baixar o dispositivo ao buscar a melhor posição) pode alterar constantemente a forma com que os símbolos sejam percebidos modificando todo contexto de análise. Para minimizar esse problema, o trabalho se baseia na solução apresentada por Stanek \& Friedmannova (2010), que utilizaram imagens estáticas do espaço externo com a sobreposição de símbolos simulando um sistema de RA.

A área de estudo do experimento foi o Centro Politécnico da Universidade Federal do Paraná (UFPR), localizado na cidade de Curitiba, estado do Paraná, Brasil. O conjunto de edificações utilizadas nos testes abriga os cursos de engenharia, física e arquitetura (Figura 4), limitando-se a uma estrutura formada por blocos que abrigam atividades de ensino e pesquisa, perfazendo um complexo de dois andares. Os blocos contêm diversos pontos de referência por ex.: equipamentos de incêndio, banheiros, escadas - que serviram como base para a construção do teste.

A escolha dos pontos de referência teve como base trabalhos de dissertação desenvolvidos no programa de Pós-graduação em Ciências Geodésicas por Antunes (2016) e Sarot (2015), que aplicaram modelos de mapeamento e navegação no espaço indoor na área do Centro Politécnico da UFPR. Os pesquisadores realizaram testes para determinar os pontos de referência do espaço indoor das edificações, sendo cada imagem capturada de modo que os pontos em cada cena pudessem ser observados em diferentes posições, pontos esses que foram selecionados para posterior criação da representação de um sistema simulado de RA. Uma préseleção das imagens foi realizada com base na qualidade dos símbolos e da iluminação ambiente. Levando em consideração as duas pesquisas, a Tabela 1 apresenta os pontos de referência selecionados, e o grau de incidência (existência e distribuição nos blocos) - referente a área de estudos. 


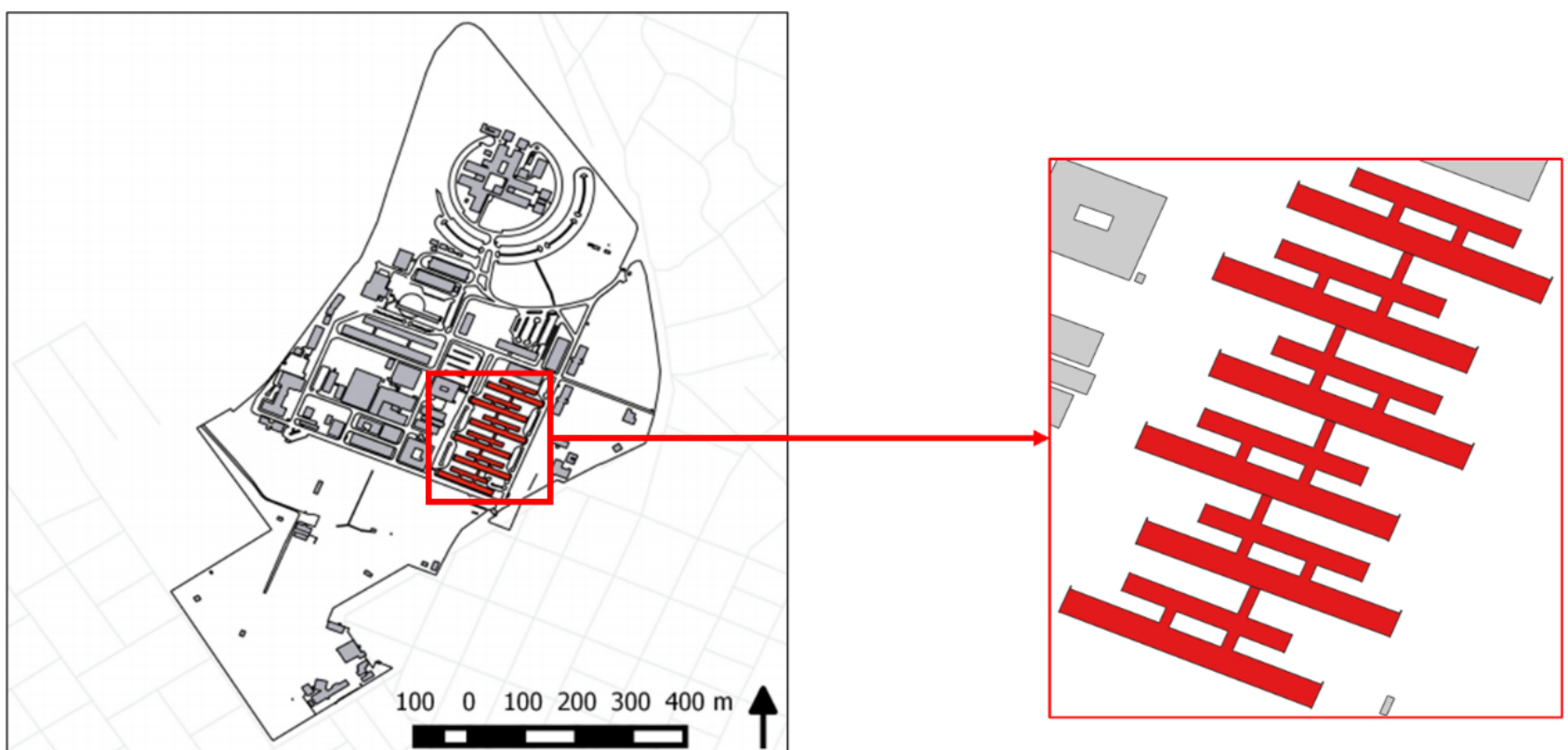

Figura 4 Centro Politécnico da Universidade Federal do Paraná.

$\mathrm{Na}$ área de estudo, há muitas repetições de pontos de referência de um ambiente para outro, existindo pouca diversidade, o que dificulta a navegação/orientação dos usuários, devido à ausência de familiaridade ou falta de uma rotina de frequência no equipamento em questão. A decisão tomada de se colocar dois pontos de referência representados por símbolos pictóricos em cada cena (imagem construída) reflete justamente a repetição e a baixa variação de pontos em cada ambiente.

Por esse motivo, a partir de um conjunto de pontos de referência presentes nos trabalhos de Sarot (2015) e Antunes (2016), o atual estudo escolhe os seguintes pontos para o experimento: banheiro masculino, escada, elevador, saída e rampa de acesso, os quais são representados por símbolos pictóricos trabalhados em um contexto de simulação em RA. Ressalta-se que nem todos os pontos de referência tem a mesma incidência de símbolos, porém é comum

Tabela 1 Pontos de referência selecionados dos trabalhos de Sarot (2015) e Antunes (2016)

\begin{tabular}{lc}
\hline Pontos de Referência & Grau de Incidência \\
\hline Banheiro masculino & Alta \\
Escada & Alta \\
Elevador & Alta \\
Saída & Alta \\
Rampa de acesso & Alta \\
\hline
\end{tabular}

que alguns desses pontos tendam a se repetir ao longo de um trajeto e no conjunto dos blocos, como é o caso das escadas de acesso para o segundo pavimento.

Assim, os símbolos pictóricos utilizados nesse estudo se baseiam em Sarot (2020), que trabalhou um conjunto de símbolos pictóricos conhecidos por pictogramas para pontos de referência aplicáveis em ambientes indoor do Centro Politécnico da UFPR. Os símbolos tem como referência a Coletânea de Normas Técnicas para símbolos gráficos: a ISO 7001, que reúne os padrões para símbolos gráficos de informação ao público, e nas determinações para o desenvolvimento de símbolos do Google Street Maps e do OpenStreetMap (Sarot 2020). O material serviu de apoio para a adaptação de um conjunto de símbolos pictóricos (Figura 5), junto a um referencial teórico composto principalmente por Forrest \& Castner (1985) e Andrade \& Sluter (2012).

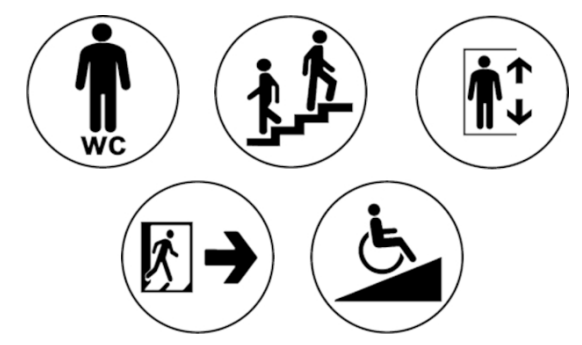

Figura 5 Símbolos pictóricos adaptados (Sarot 2020). 
Os símbolos selecionados foram projetados para representações bidimensionais, não sendo consideradas as especificidades do contexto da RA. Uma das principais alterações realizadas nos símbolos está relacionado a remoção do fundo branco que as compõem. Os estudos sobre símbolos pictóricos em RA, até então, aplicam símbolos com preenchimento de fundo (branco) ou com fundo semitransparente (Halik 2012; Halik \& MedyńskaGulij 2016), seguindo as orientações de Stanek \& Friedmannova (2010). Contudo, a tela dos dispositivos móveis, como, por exemplo, os smartphones em sua maioria, é relativamente pequena. Isto faz com que os elementos virtuais se apresentem desproporcionais em relação à tela do dispositivo, não permitindo a visualização das características (visuais) do ambiente real. Procedeuse então com a aplicação da total transparência do fundo, deixando apenas as formas que compõem a imagem e permitindo que os aspectos do espaço concreto-real possam ser observados. Os símbolos são apresentados em cor preta, seguindo a metodologia apresentada por Halik \& MedyńskaGulij (2016), em que os autores aplicaram a cor preta para analisar a percepção de símbolos pictóricos em diferentes fundos no contexto da RA.

Como a realidade graficamente representada possui uma configuração de cores diversas e não arbitrárias, problemas relacionados ao contraste poderiam ser ocasionados. Para evitar problemas de baixo contraste a tonalidade dos símbolos foi ajustada para $R=0, G=0, B=0$, aplicando-se maior contraste em suas feições em relação ao fundo. Do mesmo modo, as molduras também foram ajustadas para se adequar à relação figura-fundo que deve ser preservada em RA.

O tamanho dos símbolos foi determinado com base no padrão de tamanho de ícones para o sistema Android do Google (Figura 6). Os tamanhos padronizados contemplam diferentes tamanhos de tela e diferentes dispositivos que usam sistema Android. Para dispositivos móveis, sobretudo para representações cartográficas, deve -se considerar a orientação de autores como Montez (2012), que aborda essa questão para representações de RA. Para o teste realizado aplicou-se três variações diferentes para telas restritas dos dispositivos móveis: xxhdpi, equivalente a 144 px, hdpi,

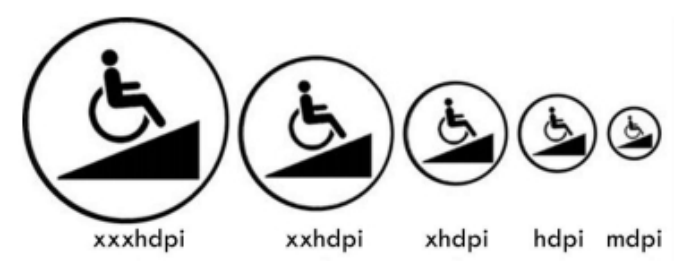

Figura 6 Tamanho de ícones para o sistema Android (Adaptado de Google, 2019). equivalente a $72 p x$ e mdpi, equivalente a 48 px, conforme destacado na Figura 6.

O trabalho de Livingston et al. (2012) investigou a percepção e a acuidade visual em sistemas de RA identificando que o contraste define a capacidade do observador em discernir as diferenças de luminosidade na imagem que, associado ao tamanho do símbolo, determina o reconhecimento nesses sistemas. Portanto, quanto maior a luminosidade no espaço representado na imagem, maior o seu contraste, do mesmo modo espaços com mais sombras e menor luminosidade possuem baixo contraste. Com base nessa determinação foram selecionadas três imagens representando espaços distintos, os quais são chamados de cenários.

No cenário 1 , os símbolos pictóricos de saída e elevador são apresentados em um espaço com alto contraste e poucas sombras; o cenário 2 os símbolos pictóricos de escada e banheiro masculino estão dispostos em um espaço com contrastes médios com iluminação ambiental moderada e algumas sombras; o cenário 3 apresenta os símbolos pictóricos de rampa de acesso e escada em um espaço de baixo contraste, pouca iluminação do ambiente e muitas sombras. Cada cenário apresentou os símbolos posicionados próximos aos seus equivalentes no espaço real. Com as dimensões definidas entre xxhdpi, hdpi e mdpi, foram admitidas três variações de tamanho entre dois símbolos, A e B. Deste modo, cada cenário foi formado por um conjunto de três imagens nas quais os símbolos possuem as seguintes variações de tamanho entre si: $\mathrm{A}>\mathrm{B}, \mathrm{A}=\mathrm{B}$ e $\mathrm{A}<\mathrm{B}$ (Figura 7).

No contexto do espaço indoor com os símbolos em RA, o experimento propõe que o participante identifique o símbolo mais próximo em relação ao outro em uma representação estática. Em outras palavras, o usuário tinha disponível uma sequência de cenas compostas por dois símbolos pictóricos cada, sendo acompanhado de um questionamento que, ao final, permite avaliar se consegue estimar a relação de distância entre os pontos especificados.

Por se tratar de uma edificação pública com acesso livre de pessoas, a pesquisa foi direcionada a um grupo muito heterogêneo. A pesquisa contou com um formulário digital distribuído em diversos canais de comunicação.

O questionário foi dividido em duas partes: características do usuário, dimensionalidade dos símbolos e, posterior questionário de finalização.

$\mathrm{Na}$ primeira parte faz-se um breve perfil do participante, com a coleta de informações gerais como o grau de instrução e experiência no uso de mapas. Essas características serviram de base para identificar se existe algum tipo de relação entre o grau de instrução e a capacidade de estimar distâncias através dos símbolos representados. 


\section{CENÁRIO 1}
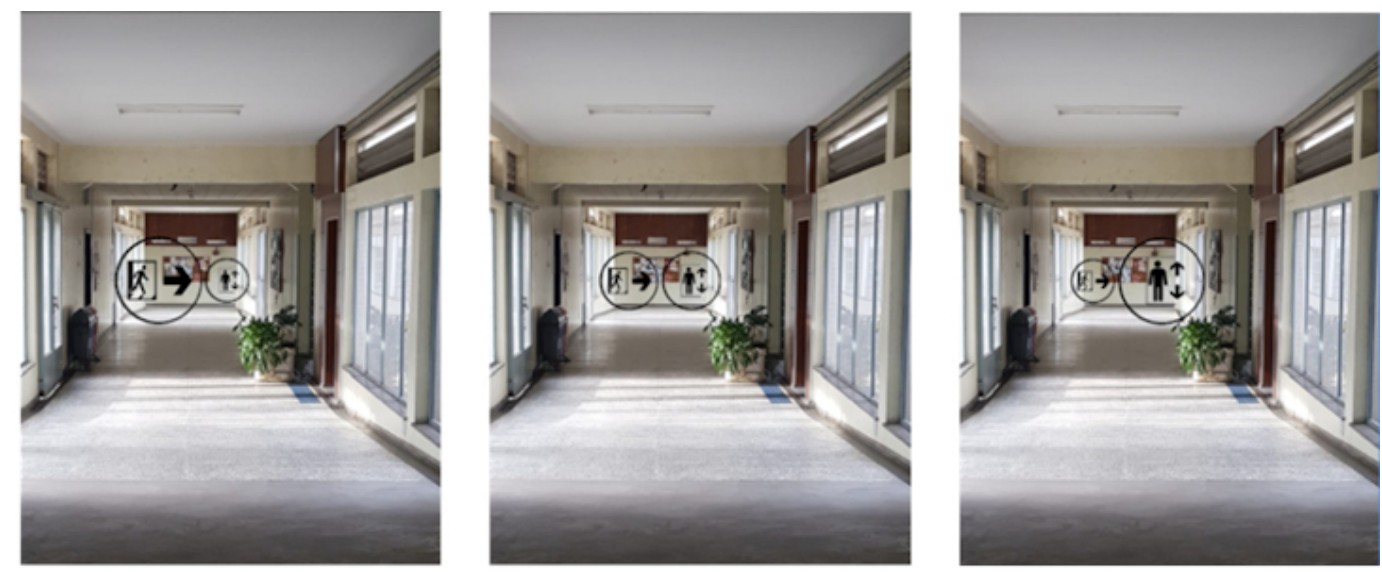

CENÁRIO 2
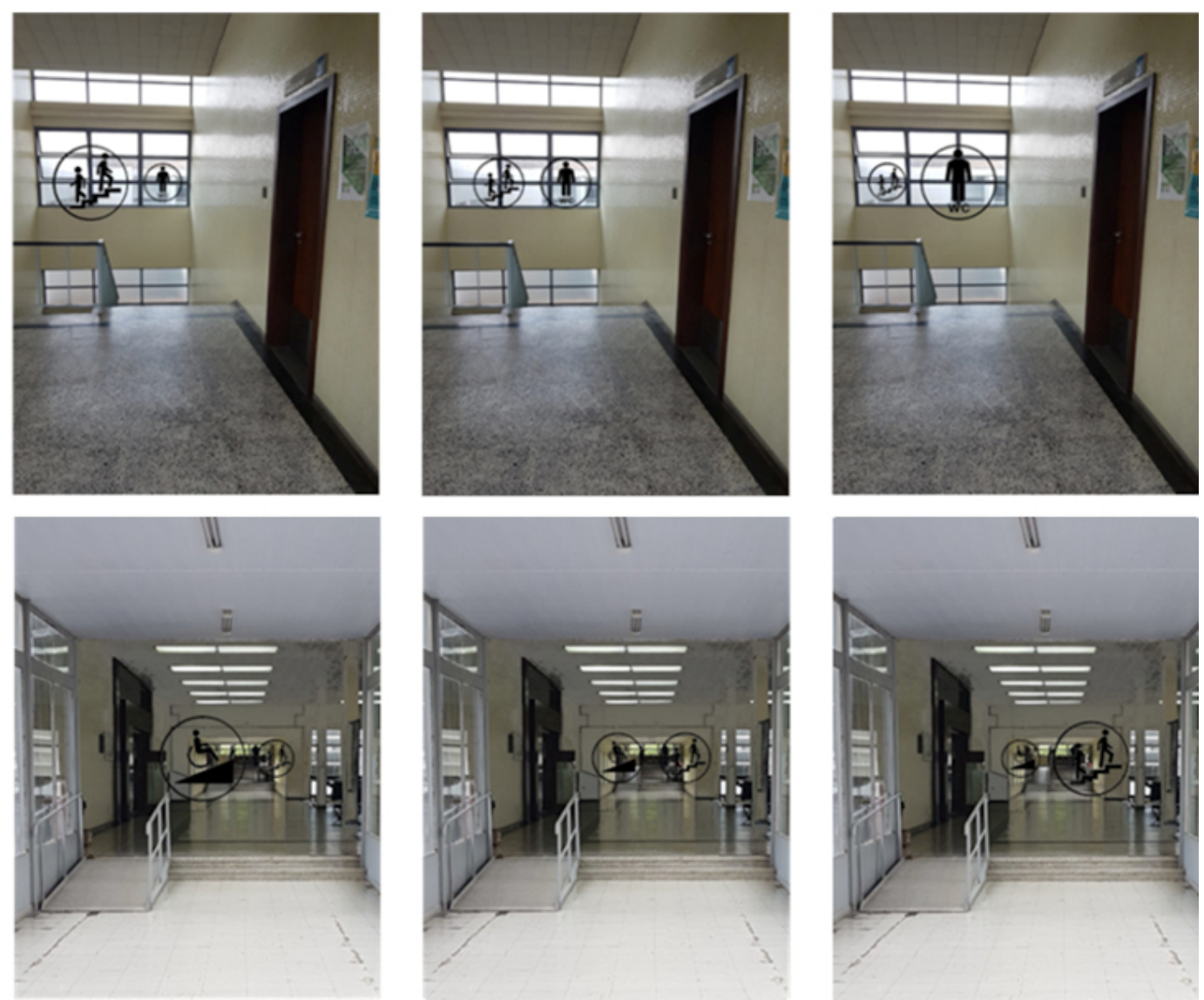

Figura 7 Cenários e distribuição dos símbolos pictóricos.

A segunda parte, refere-se a questão da percepção de proximidade e/ou distanciamento dos pictogramas. Nesta etapa, apresenta-se ao o usuário a uma sequência de nove questões com imagens representando uma imagem do espaço interno dos blocos da área de estudo. Cada questão apresentava uma imagem com a dupla de símbolos pictóricos posicionados próximos aos pontos de referência e em três cenários distintos. Na Figura 8 os símbolos relacionados à rampa e escada estão posicionados próximos dos seus equivalentes no mundo real. 


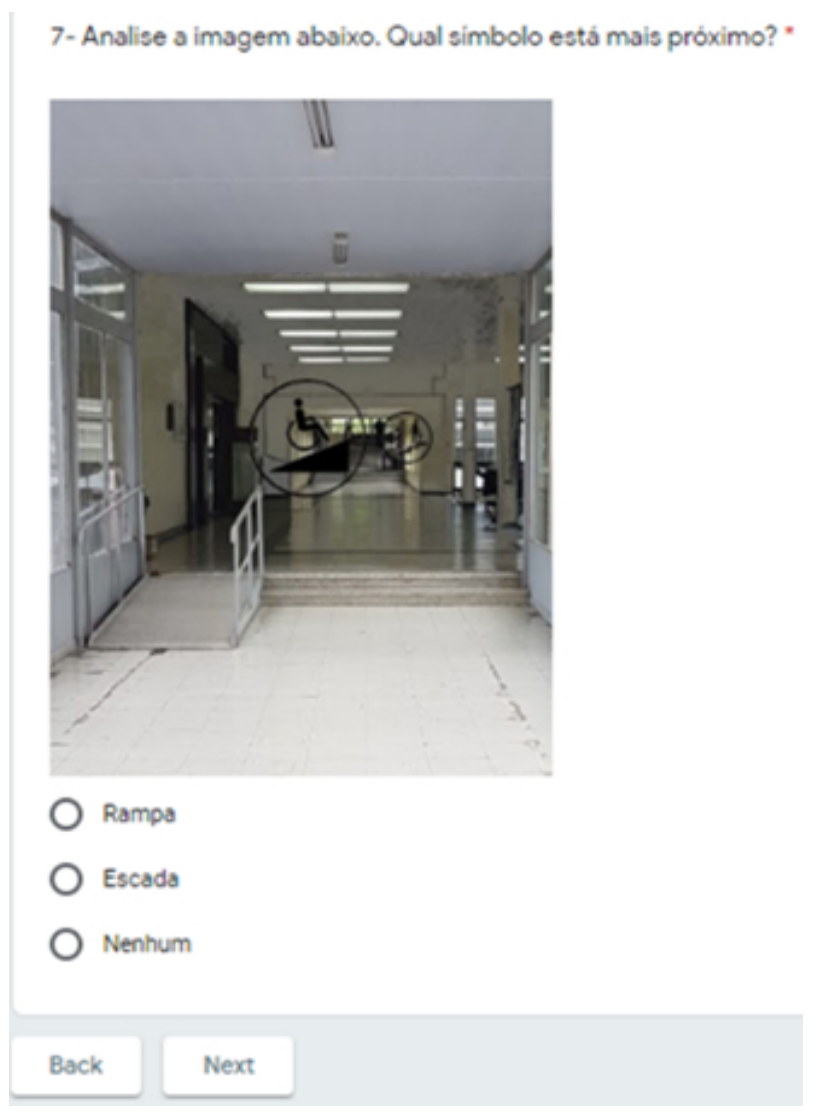

Figura 8 Exemplo de questão de avaliação da dimensionalidade.

As questões foram apresentadas de forma alternada aos participantes, não permitindo que houvesse sequencialidade nas imagens referentes a um mesmo cenário evitando qualquer tipo de dedução no ordenamento. Desta forma, no segundo questionário (percepção de proximidade e/ou distanciamento dos símbolos) as questões foram apresentadas conforme o ordenamento do Tabela 2.

Em todas as questões o participante foi apresentado ao seguinte questionamento: "Analise a imagem abaixo. Qual símbolo está mais próximo?”. Três alternativas foram apresentadas como possibilidades de resposta, o nome dos símbolos dispostos na cena e a opção "nenhum". A pergunta aplicada induz o participante a buscar o símbolo mais próximo ao invés da feição na cena representada.

O cenário 1 foi aplicado nas questões 6, 8 e 11, com os símbolos de saída e elevador, em que o ambiente é iluminado com alto contraste em relação a simbologia, adquirindo clareza em sua representação. $\mathrm{O}$ cenário 2 foi aplicado nas questões 4, 9 e 12 com os símbolos de banheiro masculino e escada em um grau de dificuldade médio, pois representa os símbolos dispostos sobre uma janela com algumas sombras. O cenário 3 foi aplicado nas questões 5 , 7 e 10 com os símbolos de rampa de acesso e escada, com
Tabela 2 Organização das questões para a segunda parte do questionário

\begin{tabular}{cc}
\hline Questões & Cenário \\
\hline 4 & 2 \\
5 & 3 \\
6 & 1 \\
7 & 3 \\
8 & 1 \\
9 & 2 \\
10 & 3 \\
11 & 1 \\
12 & 2 \\
\hline
\end{tabular}

maior nível de dificuldade pois possuía baixo contraste, muitas sombras provenientes da iluminação natural. A relação de proximidade e distanciamento dos símbolos foi apresentada nos resultados, portanto, está relacionada aos símbolos na cena, e não ao ponto de referência a que se referem e que compõem o espaço real representado.

\section{Resultados}

O experimento reuniu 243 entrevistados, que aceitaram previamente os termos da pesquisa e cujas respostas foram analisadas com base nas características observadas no questionário de perfil do usuário. Os resultados possibilitaram descrever algumas tendências da percepção do dimensionamento dos símbolos. Os participantes foram caracterizados quanto ao seu nível de escolaridade (fundamental, médio, superior e pós-graduação), e experiência com mapas: baixo (uso esporádico), médio (uso moderado) e alto (uso frequente).

Quanto ao nível de escolaridade, a maior parte dos participantes, o equivalente a $35 \%$, declarou possuir curso superior, os participantes com nível médio totalizam 33\%, $28 \%$ possui pós-graduação e $3 \%$ do grupo amostral declarou possuir apenas o ensino fundamental. No que se refere à experiência com mapas, a maior parte dos participantes possui baixo nível de experiência com mapas, $72 \%$ se enquadram nesta categoria $15 \%$ possui nível médio e $13 \%$ declarou fazer uso frequente de mapas, com alta experiência. A partir das características dos entrevistados, o Figura 9 apresenta o percentual de acertos em cada questão e por cenário, entende-se como acerto a expectativa de resposta para cada questão com base na hipótese apresentada.

O cenário 1 possui elevado contraste entre os elementos do espaço representado e os símbolos, atuando como fatores determinantes na noção de distanciamento, por facilitar o processo de segregação figura-fundo. Na Figura 8, 
isso pode ser observado nas questões 6,8 e 11 , que possuem níveis de acertos regulares entre si, um comportamento que não se repete nas outras questões. O cenário 2 possui um contraste moderado com a presença de sombras que diminuem o contraste em relação ao cenário anterior, as questões 4,9 e 12 apresentam menor número de acertos. $\mathrm{O}$ cenário 3 possui menos contraste e, por consequência, maior diferença entre as questões 5,7 e 10 , apresentando maior número de acertos quando o símbolo referente à rampa de acesso aparece sobre seu referente em tamanho maior.

O resultado está alinhado aos trabalhos de Halik (2012) e Halik \& Medyńska-Gulij (2016), que desenvolveram estudos com símbolos semitransparentes em diferentes representações de fundo das figuras para áreas externas. Apesar do ambiente indoor ser naturalmente mais escuro, o fundo transparente dos símbolos proporciona um resultado semelhante ao encontrado pelos autores em que a percepção da dimensionalidade é facilitada pelo alto nível de contraste. Os espaços com iluminação moderada e, por esse motivo, considerados com contraste médio com a simbologia, apresentaram melhores resultados com símbolos com maior discrepância de tamanho, obtendo maiores erros de identificação quando os símbolos estavam posicionados perto de sombras do espaço.

A hipótese de Stanek \& Friedmannova (2010), de que símbolos de mesmo tamanho tendem a confundir a compreensão da profundidade em RA é comprovada através da análise de quase todas as questões, exceto no cenário 1 em que o fundo ressaltado pelo elevado contraste é ressaltado. Esse resultado demonstra uma diferença substancial entre a representação dos espaços indoor e os ambientes externos, por possuir características de iluminação diferentes. O relacionamento figura-fundo tem uma importância significativa na interpretação dos símbolos. Nesse sentido, as respostas referentes a uma representação com alto nível de contraste obtiveram melhores resultados; por outro lado, as colocadas sobre uma imagem com menor taxa de contraste apresentaram resultados inferiores.

A análise pelo nível de escolaridade considerou os participantes que alegaram possuir ensino fundamental, médio, superior e pós-graduação (Figura 10). Foi realizada uma análise preliminar dos dados e em seguida os mesmos foram submetidos a Análise de Variância (ANOVA) para avaliar a existência de diferença significativa entre as classes.

Os participantes que declararam ter o ensino fundamental, obtiveram pontuação mais alta ao visualizar símbolos em cenários de alto contraste, em que o contexto auxilia o processo de reconhecimento das formas e no qual a diferença no tamanho dos símbolos era maior. Os entrevistados que possuem ensino médio obtiveram resultados semelhante, exceto pelas imagens que apresentavam maior diferença entre o tamanho dos símbolos sob contraste médio e baixo. Nas duas situações, a porcentagem de acerto do grupo foi menor. Os participantes que declararam ter ou cursarem graduação e pós-graduação obtiveram resultados semelhantes em todo o teste, sem variação considerável nas questões. A Tabela 3 apresenta os resultados do teste ANOVA realizado a partir da soma de todos os grupos, a significância adotada para este teste foi de 0,05. Em séries que apresentam valor de "p" menor que a significância adotada, possuem diferenças significativas.

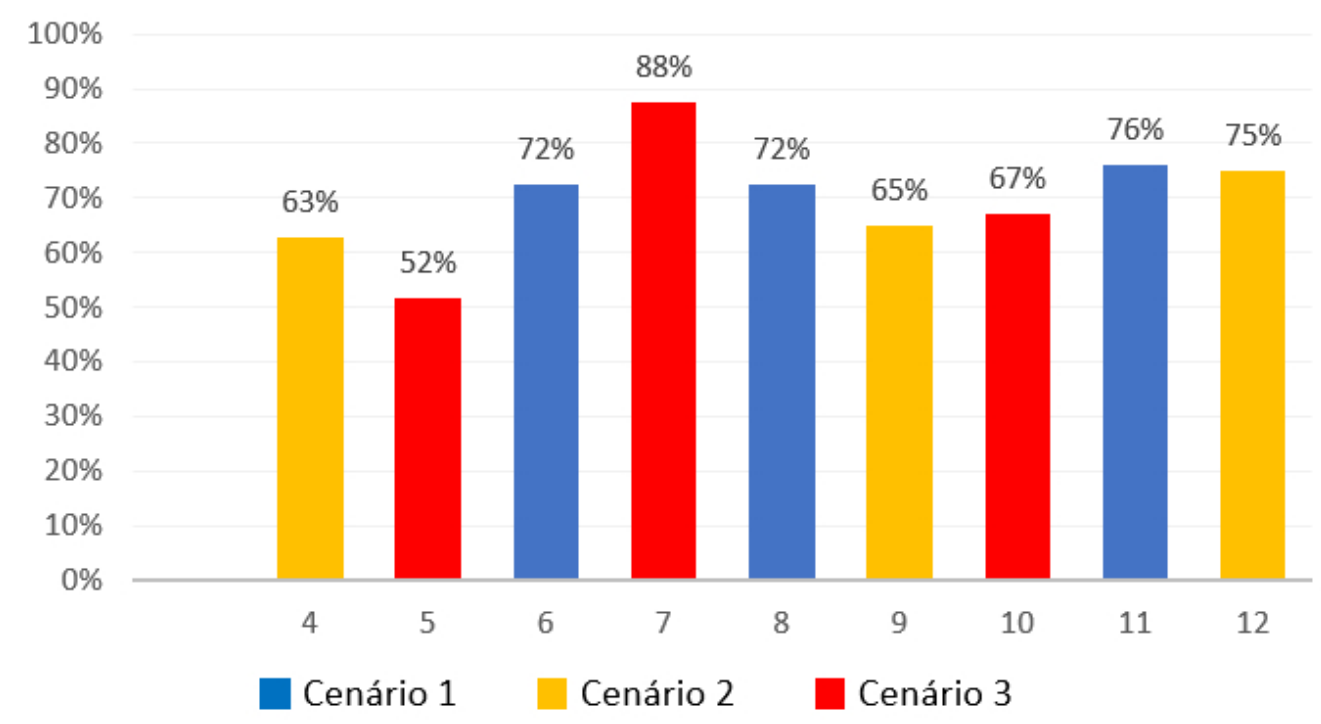

Figura 9 Total de acertos das questões por cenário. 


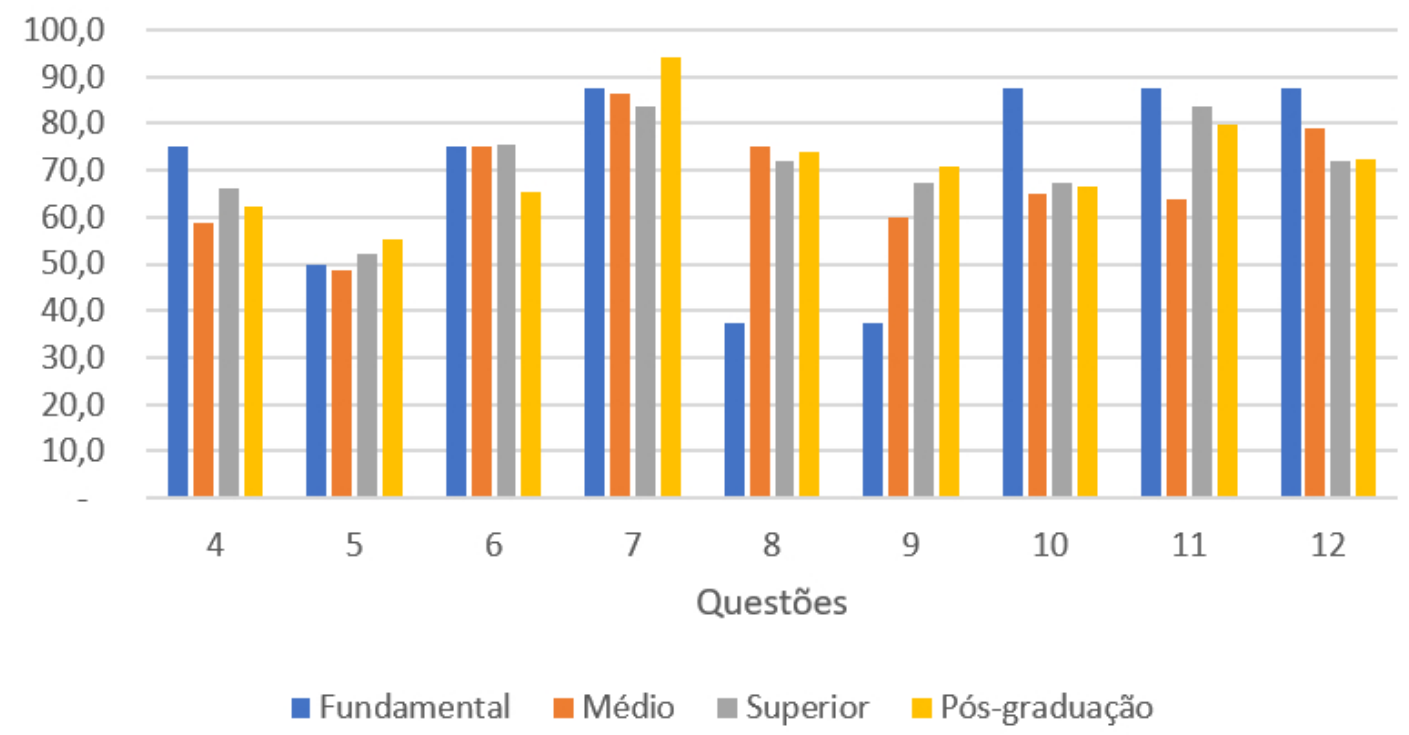

Figura 10 Acertos das questões pelo nível de escolaridade.

Tabela 3 Resultado do ANOVA para nível de escolaridade

\begin{tabular}{ccc}
\hline \multicolumn{3}{c}{ Análise de Variância - $\mathbf{\alpha} \mathbf{5 \%}$} \\
\hline \multicolumn{3}{c}{ Escolaridade } \\
\hline Questões & Razão F & Valor-p \\
\hline 4 & 1,37 & 0,252 \\
5 & 0,20 & 0,895 \\
6 & 0,83 & 0,477 \\
7 & 1,37 & 0,252 \\
8 & 1,75 & 0,157 \\
9 & 1,63 & 0,184 \\
10 & 0,55 & 0,646 \\
11 & 3,61 & 0,014 \\
12 & 0,62 & 0,601 \\
\hline
\end{tabular}

Enquanto o teste Tukey (que compara médias duas a duas) foi aplicado para identificar quais grupos possuíam as diferenças significativas ressaltadas na questão 11 (Tabela 3). Observou-se que os participantes que alegaram possuir nível superior e aqueles que possuíam nível médio, foram os grupos com maior diferença, com $83,8 \%$ e $63,8 \%$ respectivamente. Neste caso específico, refere-se ao cenário com alto contraste e igualdade de tamanho dos símbolos, conforme apontado anteriormente em Stanek \& Friedmannova (2010).

Também foram avaliados a quantidade de acertos pelo nível de experiência com mapas. Os participantes que alegaram possuir experiência no uso de mapas tiveram quantidade de acertos acima de $60 \%$ nas questões enquanto aqueles que afirmaram não possuir experiência no uso de mapas possuíram uma variação a partir de $35 \%$ de acertos.

A pontuação final baseada no nível de experiência com mapas é dividida entre alta, média e baixa. A Figura 10 apresenta uma resposta equilibrada entre o uso alto e médio de mapas. Nos cenários 4 e 5 , os usuários que declararam ter experiência média obtiveram uma pontuação melhor que os demais usuários. Os participantes que declararam ter níveis mais baixos de uso do mapa obtiveram o menor percentual de acertos, chegando a $45 \%$ no cenário 2 (Figura 11).

Os valores mais altos representados na Figura 10 pertencem aos grupos que alegaram possuir nível médio e alto no uso dos mapas. É possível observar que ocorre um processo decrescente entre o nível alto e médio em relação ao nível baixo. Isso corrobora com os estudos apresentados em Ooms, De Meyer \& Fack (2015), que já demonstravam uma tendência nesse sentido.

A Tabela 4 também apresenta a análise com base no ANOVA para a quantidade de acertos pelo nível de experiência com mapas. Pode-se perceber que, nas questões 4 e 7 os valores não ficam abaixo do nível de significância, ou seja, acima de 0,05 , o que indica a existência de diferenças significativas entre as classes. Dessa forma, também foi aplicado o teste Tukey para avaliar onde encontram-se as diferenças. Observou-se que as maiores variações ocorreram entre o grupo que declara possuir baixo nível de uso de mapas e o grupo que declara possuir alto nível de uso de mapas. É possível observar que a experiência no uso de mapas aparece como elemento significativo na interpretação. 


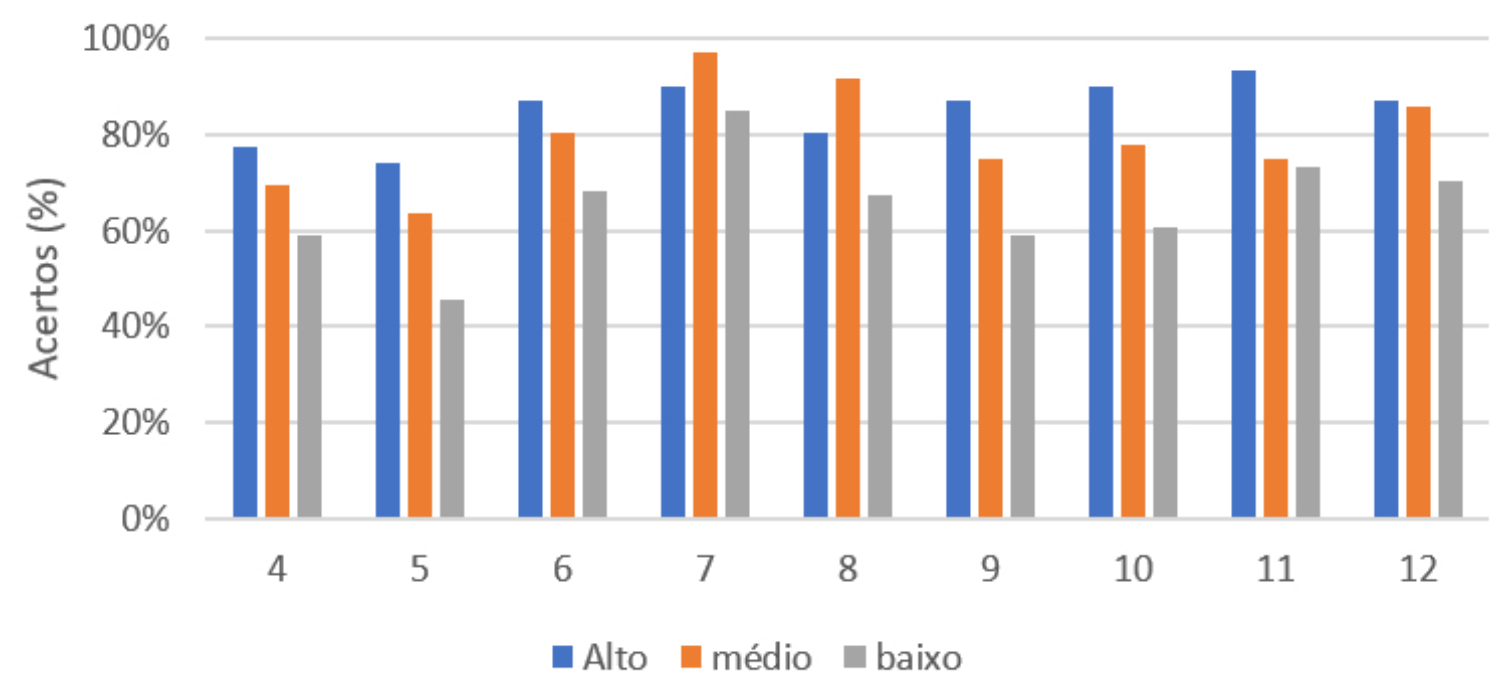

Figura 11 Total de acertos das questões pelo nível de experiência com mapas.

Tabela 4 Resultado do ANOVA para uso de mapas

\begin{tabular}{ccc}
\hline \multicolumn{3}{c}{ Análise de Variância - $\mathbf{\alpha} \mathbf{5 \%}$} \\
\hline \multicolumn{3}{c}{ Uso de mapas } \\
\hline Questões & Razão F & Valor-p \\
\hline 4 & 2,11 & 0,123 \\
5 & 5,78 & 0,004 \\
6 & 3,10 & 0,047 \\
7 & 2,11 & 0,123 \\
8 & 4,67 & 0,010 \\
9 & 5,66 & 0,004 \\
10 & 6,56 & 0,002 \\
11 & 3,03 & 0,050 \\
12 & 3,41 & 0,035 \\
\hline
\end{tabular}

Observou-se que os cenários com contraste baixo entre os elementos do espaço representado e os símbolos, são determinantes na noção de distanciamento, ainda que os símbolos apresentem elevada diferença de tamanho. Símbolos de mesmo tamanho tendem a confundir na compreensão da profundidade, em quase todos os cenários, os conjuntos de símbolos com o mesmo tamanho apresentaram problemas na sua identificação. Os espaços com iluminação moderada e, por esse motivo, considerados com contraste médio com a simbologia, apresentaram melhores resultados com símbolos com maior discrepância de tamanho, obtendo maiores erros de identificação quando os símbolos estavam posicionados perto de sombras do espaço. Por outro lado, os espaços com alto contraste possuíram bons resultados na determinação da distância entre os símbolos em todas as situações. Observou-se, contudo, que os participantes, em sua maioria, conseguiram identificar os símbolos mais próximos e mais distantes de si.

Os testes demonstraram que os símbolos pictóricos construídos com base nas leis da Gestalt e com alteração em suas dimensões, transmitem a noção de profundidade e distanciamento em um contexto de RA simulada. A construção de um sistema de RA para dispositivos móveis e voltados para o ambiente indoor deve considerar o dimensionamento dos pixels como taxonomia de pistas em vista egocêntrica, sendo potencialmente aplicáveis a sistemas de RA. Os resultados obtidos no experimento indicam que a maioria dos participantes da pesquisa não enfrentou desafios para estimar a variação da distância entre os símbolos apresentados, estimando o distanciamento entre os símbolos conforme o esperado.

Os participantes demonstraram que, para uma representação em RA de um espaço interno, a avaliação da dimensionalidade do símbolo pode ser transferida para o entendimento espacial relacionado ao distanciamento entre o usuário e os pontos de referência. A relação entre o tamanho dos símbolos e a proximidade e/ou distanciamento, conforme proposto por Kraak (1988), é aplicável ao contexto proposto e preserva a relação entre tamanho e distanciamento em que os símbolos maiores representam elementos mais próximos ao usuário e símbolos menores representam elementos mais distantes dos usuários.

Além disso, evidenciou-se que os usuários não encontraram dificuldades na interpretação dos formatos dos símbolos quando o contraste foi elevado, no entanto o mesmo não se aplica ao reconhecer o tamanho dos símbolos em salas escuras. Isso indica que, em um sistema 
de navegação, o projeto dos símbolos deve considerar os aspectos relacionados à variação da iluminação do ambiente, que podem interferir no processo de reconhecimento de formas, ao projeto de símbolos $2 \mathrm{D}$ e sua adequação para RA e a dimensionalidade. A aplicação de um pano de fundo totalmente transparente aos símbolos pictóricos pode ter levado à questão identificada, porém é um elemento que surge como questão para estudos futuros, a fim de ser investigado e realizado em uma comparação no processo interpretativo e na coleta de dados espaciais com base em símbolos sólidos contra fundos transparentes.

Outra característica demonstrada no experimento relacionada à cor dos símbolos, levando em consideração as leis da Gestalt, afirma que o preto tem uma visão melhor sobre o fundo branco. Mais testes com outras cores com símbolos sob fundo transparente são necessários, devido à complexidade e variação da informação presente em um ambiente indoor, principalmente por causa das sombras.

Observou-se que a compreensão dos símbolos pictóricos desenvolvidos com base nas leis da Gestalt, e voltados para a representação de feições em espaços indoor, possui características similares ao que têm sido apresentado por Basiri et al. (2017); Halik (2012); Halik \& Medyńska-Gulij (2016); e Stanek \& Friedmannova (2010). As complexidades dos espaços indoor, que os colocam em uma categoria singular da representação cartográfica (Nossum 2013), são apresentadas nesse trabalho como uma análise inicial e fundamental para o aprofundamento das questões relacionadas à aplicação de símbolos pictóricos em RA.

\section{Conclusão}

Os resultados obtidos no teste demonstram que a relação de distanciamento ou proximidade podem ser obtidas em sistemas de RA através de uma simbologia bidimensional, desenvolvida com base nas leis da Gestalt e adaptada às especificidades do espaço indoor. A adequação da simbologia desenvolvida nos aspectos relacionados à moldura e transparência de fundo, contribuíram para o processo de segregação figura-fundo, possibilitando a percepção do tamanho relativo entre os símbolos pictóricos apresentados.

Em sua maioria, os entrevistados conseguiram compreender os recursos visuais de profundidade transmitidos e identificar símbolos pictóricos mais próximos, por possuir maior tamanho, em relação aos símbolos pictóricos mais distantes, que possuem menor tamanho. Todavia, as alterações de contraste impostas pelo ambiente indoor atuam como um limitador, pois as variações de luminosidade e de contexto não são controláveis. A relação de profundidade é percebida com maior facilidade quando associada a um contraste alto ou moderado em relação ao fundo e com dois ou mais símbolos pictóricos representados.

O teste foi realizado com imagens estáticas a partir de representações gráficas (pictóricas), o que impossibilitou a interação simulada entre o entrevistado e espaço indoor com RA. O aspecto (estático) é um limitador da pesquisa, pois ao ver os símbolos em diferentes perspectivas da vista egocêntrica pode ter influência na identificação do tamanho relativo. Ressalta-se ainda que mesmo não sendo um fator de análise do trabalho, o recurso movimento pode influenciar a percepção de distâncias em contraponto às imagens estáticas, sendo uma sugestão para aplicações futuras.

O projeto de símbolos é outro fator determinante para a compreensão da simbologia e está diretamente relacionado à implementação das leis da Gestalt. O projeto deve ser adaptado às características do espaço real-concreto representado, implementando alterações estruturais na simbologia, como alteração de tamanho, contraste, remoção do fundo branco e iluminação do ambiente, que possam permitir a melhor compreensão da forma. O estudo demonstrou que o processo é viável, mas requer mais detalhes no projeto dos símbolos, ou seja, o desenvolvimento de uma simbologia específica e direcionada ao contexto da RA.

Em trabalhos futuros, sugere-se que estudos similares com sistemas de RA em tempo real possam ser aplicados para avaliar o impacto do movimento na percepção do distanciamento com a variação do tamanho dos símbolos. Isto porque, o estudo aqui apresentado é um ponto de partida para o desenvolvimento novos trabalhos relacionados às representações cartográficas aplicadas em espaços internos.

\section{Agradecimentos}

Os autores agradecem à Capes pela concessão da bolsa de doutorado e ao CNPq pela Bolsa Produtividade em Pesquisa (processo 310312/2017-5).

\section{Referências}

Al Delail, B., Weruaga, L., Zemerly, M.J. \& Ng, J.W. 2013, 'Indoor Localization and Navigation Using Smartphones Augmented Reality and Inertial Tracking', IEEE International Conference On Electronics, Circuits, and Systems, pp. 929-32.

Andrade, A.F.A. 2014, 'Gestalt na avaliação da simbologia pictórica com base em tarefas de leitura de mapas', PhD. Thesis, Universidade Federal do Paraná.

Andrade, A.F.\& Sluter, C.R. 2012, 'Avaliação De Símbolos Pictóricos Em Mapas Turísticos'. Boletim de Ciências Geodésicas, vol. 18, no. 2, pp. 242-261. 
Antunes, A.P. 2016, 'Avaliação de pontos de referência com uso de QR-code para posicionamento em ambiente indoor', Master Thesis, Universidade Federal do Paraná.

Basiri, A., Lohan E.S., Moore T., Winstanley A., Peltola P., Hill C., Amirian P. \& Silva P.F. 2017, 'Indoor location based services challenges, requirements and usability of current solutions'. Computer Science Review, vol. 24, pp. 1-12.

Biehler, D.D. \& Simon, G.L. 2011, 'The great indoors - Research frontiers on indoor environments as active political - ecological spaces'. Progress In Human Geography, vol. 35, no. 2, pp. 172-92.

Chen, J. \& Keith C. 2020, 'Indoor cartography', Cartography and Geographic Information Science, vol. 47, no. 2, pp. 95-109.

Dick, M.E., Gonçalves, B.S., Pereira, A.T.C. \& Vieira, M.L.H. 2016, 'A influência dos dispositivos portáteis de leitura no design do livro digital'. Blucher Design Proceedings, pp. 761-68.

Fiori, S.R. \& Lucena, R.W.A. 2020, 'O uso da comunicação visual na Geografia: a ilustração nos ambientes escolar, acadêmico e profissional', Caminhos De Geografia, no. 75, vol. 21, pp 117-36.

Forrest, D. \& Castner, H.W. 1985, 'The design and perception of point symbols for tourist maps'. The Cartographic Journal, vol. 22, no. 1, pp. 11-9.

Google ARCore Developers 2019, Google for Startups Accelerators: Women Founders, viewed 19 Jun. 2019, <https:// developers.google.com/>.

Haeberling, C. 2002, '3D-map presentation: A Systematic Evaluation of Important Graphic Aspects', ICA Mountain Cartography Workshop, p. 1-11.

Halik, Ł. 2012, 'The analysis of visual variables for use in the cartographic design of point symbols for mobile Augmented Reality applications'. Geodesy and Cartography, vol. 61, no. 1, pp. 19-30.

Halik, Ł. \& Medyńska-Gulij, B. 2016, 'The Differentiation of Point Symbols using Selected Visual Variables in the Mobile Augmented Reality System'. The Cartographic Journal, vol. 54 no. 2 , pp. 147-56.

Hogg, J. L. 2012, 'Cognitive design considerations for augmented reality', EEE International Conference On E-Learning, E-Business, Enterprise Information Systems, and E-Government, pp. 207-14.

Ilkovičová, L', Erdélyi, J. \& Kopáčik, A. 2014, 'Positioning in indoor environment using QR codes', INGEO 2014 - 6th International Conference on Engineering Surveying, pp. 117-22.

Korpi, J. \& Ahonen-Rainio, P. 2010, 'Cultural constraints in the design of pictographic symbols'. The Cartographic Journal, vol. 47, no. 4, p. 351-59.

Korpi, J. \& Ahonen-Rainio, P. 2015, 'Design Guidelines for Pictographic Symbols -Evidence from Symbols Designed by Students', Conference Paper Eurocarto, pp. 1-19.

Kraak, M.J. 1988, 'Computer-assisted cartographical threedimensional imaging techniques', PhD. Thesis. Delft University Press.
Kubíček, P., Šašinka Č., Stachoň Z., Herman L., Juřík V., Urbánek T. \& Chmelík J. 2017, 'Identification of altitude profiles in 3D geovisualizations - the role of interaction and spatial abilities', International Journal of Digital Earth, vol. 12, no. 2, pp. 156-72.

Li, Y., Zhu, Q., Fu, X., Feng, B., Liu, M., Zhang, J. \& Yang, W. 2019, 'Semantic Visual Variables for Augmented Geovisualization'. The Cartographic Journal, vol. 57, no. 1, pp. 43-56.

Liu, K., Motta, G., Ma, T., \& Guo, T. 2016, 'Multi-floor Indoor Navigation with Geomagnetic Field Positioning and Ant Colony Optimization Algorithm'. Ieee Symposium On Service - Oriented System Engineering (Sose), pp. 314-23.

Livingston M.A., Gabbard J.L., Swan J.E., Sibley C.M. \& Barrow J.H. 2012, 'Basic perception in head-worn augmented reality displays' in L. Alem \& M. Livingston (eds), Human Factors in Augmented Reality Environments, New York, pp. 35-65.

Maceachren, A.M. 1995, How Maps Work: representation, visualization, and design, Guilford Press, New York.

Massironi, M. 2010, Ver pelo desenho - aspectos técnicos, cognitivos, Comunicativos, Edições 70, Lisboa.

Montez, E.J.S. 2012, 'Visualização de pontos de interesse em dispositivos móveis usando realidade aumentada', Master Thesis, Universidade de Lisboa.

Naver 2020, Augmented Reality - Naver Labs Europe, viewed 15 Oct. 2020. $<$ https://europe.naverlabs.com/tag/augmentedreality $>$.

Nossum, A.S. 2013, 'Developing a framework for describing and comparing indoor maps', The Cartographic Journal, vol. 50, no. 3, p. 218-24.

Ooms, K., De Meyer, P. \& Fack, V. 2015, 'Listen to the the Map User: Cognition, Memory, and Expertise', The Cartographic Journal, vol. 52, no. 1, pp. 3-19.

Pixabay 2019, 'Restroom Public Restroom Rest room Ladies' Restroom', viewed 22 Oct. 2020, <https://pixabay.com/ vectors/restroom-public-restroom-rest-room-99226/>.

Rantakari, J., Väyrynen, J., Colley, A. \& Häkkilä, J, 2017, 'Exploring the design of stereoscopic 3D for multilevel maps', 6th Acm International Symposium On Pervasive Displays, pp. 1-6.

Santil, F.L. de P. 2008, 'Análise da percepção das variáveis visuais de acordo com as leis da Gestalt para representação cartográfica', PhD. Thesis, Universidade Federal do Paraná.

Sarot, R.V. 2015. 'Avaliação de mapas indoor para dispositivos móveis para auxílio à tarefa de orientação', Master Thesis, Universidade Federal do Paraná.

Sarot, R.V. 2020. 'Determinação dos elementos considerados marcos de referência em ambientes indoor para auxílio a navegação com uso de sistema de navegação indoor', PhD. Thesis, Universidade Federal do Paraná.

Schabus, S., Scholz, J. \& Lampoltshammer, T.J. 2015, 'A Space in a Space - Connecting Indoor and Outdoor Geography', 18th Agile International Conference On Geographic Information Science, pp. 1-5.

Stanek, K. \& Friedmannova, L. 2010, 'Cartographically Augmented Reality', The 3rd Isde Digital Earth Summit- 
Digital Earth in the Service of Society: Sharing Information, Building Knowledge - Proceedings, pp. 1-9.

Vanclooster, A., Van De Weghe, N. \& De Maeyer, P. 2016, 'Integrating Indoor and Outdoor Spaces for Pedestrian Navigation Guidance - A Review'. Transactions in Gis, vol. 20, no. 4, pp. 491-525.
Wang, Z. \& Niu, L.A. 2018, 'Data Model for Using OpenStreetMap to Integrate Indoor and Outdoor Route Planning'. Sensors, vol. 18 , no. 7 , p. 2100.

Wong, W. 2001, Princípios de forma e desenho, Martins Fontes, São Paulo.

\section{Como citar:}

Gomes, J.V.P., Delazari, L.S. \& Schmidt, M.A.R. 2021, 'Avaliação do Dimensionamento de Símbolos Pictóricos em Mapas Indoor com Perspectiva Egocêntrica para Aplicação em Sistemas de Realidade Aumentada', Anuário do Instituto de Geociências, vol. 44: 36117. https:/l doi.org/10.11137/1982-3908_2021_44_36117 